\title{
ANALYSE WICHTIGER VORBESTEHENDER RISIKOFAKTOREN BEI PATIENTEN MIT COVID-19-DIAGNOSE IN AMAPÁ, AMAZÔNIA, BRASILIEN
}

\section{ORIGINALER ARTIKEL}

OLIVEIRA, Arthur Aires de ${ }^{1}$, MOREIRA, Danilo José Silva², PIMENTEL, Jhon Allyson Sena ${ }^{3}$, COSTA, Pedro Henrique de Magalhães ${ }^{4}$, DIAS, Claudio Alberto Gellis de Mattos $^{5}$, ARAÚJO, Maria Helena Mendonça de ${ }^{6}$, OLIVEIRA, Euzébio de ${ }^{7}$, DENDASCK, Carla Viana ${ }^{8}$, SOUZA, Keulle Oliveira da ${ }^{9}$, FECURY, Amanda Alves ${ }^{10}$

\section{OLIVEIRA, Arthur Aires de. Et al. Analyse wichtiger vorbestehender} Risikofaktoren bei Patienten mit COVID-19-Diagnose in Amapá, Amazônia, Brasilien. Revista Científica Multidisciplinar Núcleo do Conhecimento. Jahrgang 06, Ed. 06, Vol. 17, S. 56-72. Juni 2021. ISSN: 2448-0959, Zugangslink: https://www.nucleodoconhecimento.com.br/gesundheit/vorbestehenderrisikofaktoren, DOI: 10.32749/nucleodoconhecimento.com.br/gesundheit/vorbestehender-risikofaktoren

\footnotetext{
${ }^{1}$ Mediziner. Föderale Universität von Amapá (UNIFAP).

${ }^{2}$ Mediziner. Föderale Universität von Amapá (UNIFAP).

${ }^{3}$ Mediziner. Föderale Universität von Amapá (UNIFAP).

${ }^{4}$ Mediziner. Föderale Universität von Amapá (UNIFAP).

${ }^{5}$ Biologe, PhD in Theorie- und Verhaltensforschung, Professor und Forscher des Chemie-Studiengangs des Instituts für Grund-, Technik- und Technologiepädagogik (IFAP) und des Graduate Program in Professional and Technological Education (PROFEPT IFAP).

${ }^{6}$ Doktor, Master in Lehr- und Gesundheitswissenschaften, Professor und Forscher des Medical Course of Macapa Campus, Federal University of Amapa (UNIFAP).

${ }^{7}$ Biologe, PhD in Tropenkrankheiten, Professor und Forscher des Physical Education Course, Federal University of Pará (UFPA).

${ }^{8}$ Theologe, PhD in Klinischer Psychoanalyse. Er arbeitet seit 15 Jahren mit wissenschaftlicher Methodik (Forschungsmethode) in der wissenschaftlichen Produktionsberatung für Master- und Doktoranden. Spezialist für Markt- und Gesundheitsforschung, Doktorand in Kommunikation und Semiotik (PUC SP).

${ }^{9}$ Sozialwissenschaftler, Facharzt für Management und Lehre der Hochschulbildung, Master-Student in Anthropischen Studien im Amazonas (UFPA).

${ }^{10}$ Biomedizin, PhD in Tropenkrankheiten, Professor und Forscher des Medical Course of Macapa Campus, Federal University of Amapá (UNIFAP), Pro-Rektor of Research and Graduate Studies (PROPESPG) der Föderalen Universität von Amapá (UNIFAP).
} 


\section{ZUSAMMENFASSUNG}

COVID-19 (Coronavirus Disease 2019) ist eine Infektion, die durch das SARS-CoV-2Virus verursacht wird, wobei erste Fälle im Dezember 2019 in Wuhan City gemeldet wurden. Es wurde festgestellt, dass bei Patienten mit Komorbiditäten ein höheres Risiko für Komplikationen und Sterblichkeit im Falle einer Infektion besteht. Die Krankheit wurde von der Weltgesundheitsorganisation im Jahr 2020 als Gesundheitsnotstand betrachtet, und in Brasilien führte das Gesundheitsministerium Maßnahmen zur epidemiologischen Überwachung von Fällen durch, die in den verschiedenen Föderativen Einheiten des Landes registriert wurden. Unter diesen geht Amapá a von einer hohen Inzidenz und Sterblichkeit aus. Diese Studie zielt darauf ab, die wichtigsten früheren Risikofaktoren bei Patienten mit COVID-19 im Bundesstaat Amapá zu analysieren. Dazu wurden die im Coronavirus-Amapá-Panel verfügbaren Sekundärdaten durch eine am 22. Mai 2020 durchgeführte Suche verwendet. Auf der Plattform wurden Daten über die Anzahl der Patienten mit COVID-19 diagnostiziert, sowie die Anzahl der Todesfälle aufgrund der Krankheit, mit einer gemeinsamen Bewertung in jedem Thema der Komorbiditäten gefunden. Nach dem Gruppieren dieser Daten in Microsoft Excel-Tabellen wurde eine quantitative und beschreibende Analyse dieser Daten durchgeführt. Im Staat wurden bis zum Tag der Abholung insgesamt 28.927 Patienten mit COVID-19 diagnostiziert. Etwa 1,31 \% (378 Fälle) hatten einen Risikofaktor, der sie anfällig für Komplikationen infolge von COVID-19 entwickeln konnte, wobei Diabetes und chronische Herzerkrankungen die Bedingungen mit der höchsten Anzahl von Datensätzen waren. Noch im Bundesstaat Amapá wurden 420 Todesfälle bei Patienten mit COVID-19 festgestellt. Davon hatten 75,71 \% (318 Fälle) zuvor Risikofaktoren für ein höheres Risiko für Komplikationen aufgrund von COVID-19, wobei Bluthochdruck und Diabetes die vorherrschenden Erkrankungen waren. Mit der Studie wurde in Amapá festgestellt, dass ein großer Teil der Bevölkerung von Patienten, die mit der Krankheit diagnostiziert wurden und die starben, eine Vorgeschichte von Risikofaktor für COVID-19 hatte. Aufgrund der Meldung von Risikofaktoren in der Todessituation, die nicht in der Anzahl der 
diagnostizierten Patienten erfasst wurden, sowie der Unspezifität, die dem Zustand der Patienten innewohnt, die durch die staatlichen Gesundheitsmechanismen überwacht werden, war es nicht möglich, eine detailliertere Analyse über den Grad der Komplikation und der Letalitätsrate durchzuführen, die durch einen bestimmten Risikofaktor und seine Assoziation mit einer Coronavirus-Infektion erzeugt wird.

Schlagworte: COVID-19, Risikofaktoren, Komorbidität.

\section{EINLEITUNG}

In der ersten Dezemberhälfte 2019 war der erste Fall einer Lungenentzündung unbekannter Herkunft in Wuhan, Provinz Hubei, China gemeldet worden. In der Folge plagte ein Ausbruch einer schweren akuten Respiatemiston (SRAG) die Provinz und machte die Weltgesundheitsorganisation (WHO) auf sich aufmerksam, die ausgehend von der Umbeschriftung ihrer Zuschreibungen erklärte, dass es sich bei der Situation um eine Epidemie handele, die durch einen neuen Subtyp des Virus der Familie Coronaviridae, Coronavirus-2, verursacht wurde, der einen öffentlichen Gesundheitsnotstand von internationaler Bedeutung (ESPII) darstellte. 2020a (COSTA et al., 2020a; FERRARI, 2020; GAUTRET et al., 2020; GUAN et al., 2020; LI et al., 2020; LIMA et al., 2020; MARTINS-CHAVES; GOMES; GOMEZ, 2020; OLIVEIRA et al., 2020; RENU; PRASANNA; GOPALAKRISHNAN, 2020; RODRÍGUEZ-COLA et al., 2020; TAY et al., 2020; ZHAl et al., 2020)

Im Februar 2020 benannte der Generaldirektor der WHO die Krankheit Coronavirus19-Krankheit (COVID-19), eine durch SARS-CoV-2 verursachte Infektion (aus dem englischen Ausdruck: Severe Acute Respiratory Syndrome Coronavirus 2), eine der sieben bekannten Coronavirus-Subtypen (COSTA et al., 2020a; RENU; PRASANNA; GOPALAKRISHNAN, 2020; HONG et al., 2020).

Aus brasilianischer Sicht wurde die COVID-19-Infektion am 3. Februar 2020 vom Gesundheitsministerium (MS) als Public Health Emergency of National Importance (ESPIN) deklariert und am 26. Februar 2020 in Brasilien erstmals bestätigt (OLIVEIRA et al., 2020; OLIVEIRA; LUCAS; IQUIAPAZA, 2020). Als Maßnahme zur Unterstützung 
des Gesundheitssystems hat das Institut für Informatik des Einheitlichen Gesundheitssystems (DATASUS) im Rahmen des Notfallplans die Virtuelle Unterstützung der Bevölkerung durch die Anwendung "Coronavírus SUS" (BRASIL, 2020a) geleistet.

In Brasilien wird der Fortschritt der Pandemie hauptsächlich von der MS durch statistische Indizes überwacht, die den Status jeder föderalen Einheit (UF) widerspiegeln, und der Staat Amapá geht von hohen epidemiologischen Indizes aus, insbesondere in Bezug auf den Inzidenzkoeffizienten von COVID-19 (BRASIL, 2020c).

Bis zum 17. Juli 2020 führte Amapá den brasilianischen Norden im Ranking des COVID-19 Inzidenzkoeffizienten pro Meldung STATE (Anzahl der bestätigten Fälle pro 100.000 Einwohner) an, deren Wert 3.637,4 betrug, gefolgt von Roraima mit 3.327,1, das wiederum vom Staat Amazonas mit 1962,0 untergeordnet wurde. Was den COVID-19 Mortalitätskoeffizienten (Anzahl der Todesfälle pro 100.000 Einwohner) betrifft, so lag Amapá auf dem 7. Platz (55,2) (BRASIL, 2020c).

Risikofaktoren beziehen sich auf alle Bedingungen, die die Immunantwort des Körpers auf eine Krankheit beeinträchtigen. So umfassen diese Faktoren sowohl Komorbiditäten, d. h. chronische Krankheiten, die zuvor installiert wurden und die gleichzeitig im Organismus des Patienten wirken, als auch Besonderheiten von Individuen, nämlich: Puerperalzustand, Rauchen, Alkoholkonsum, unter anderem (PIRES; CARVALHO; XAVIER, 2020; ENGIN; ENGIN; ENGIN, 2020; KHALIQ; PHOSWA, 2020).

Studien deuten darauf hin, dass Patienten, die mit COVID-19 infiziert sind und Komorbiditäten haben, eine höhere immunologische Anfälligkeit sowie eine höhere Tödlichkeitsrate haben, verglichen mit Patienten, die nur vom Virus betroffen sind. Zu den wichtigsten aufgeführten Komorbiditäten gehören Bluthochdruck, Diabetes, Herzerkrankungen und chronische Erkrankungen des Atmungssystems (COSTA et al., 2020a; FERRARI, 2020; MARTINS-CHAVES; GOMES; GOMEZ, 2020; RENU; PRASANNA; GOPALAKRISHNAN, 2020; YANG et al., 2020). 
Angesichts des globalen Panoramas und des Status des Bundesstaates Amapá ist es von wesentlicher Bedeutung, Analysen, Studien und Beförderungen in der wissenschaftlichen Forschung durchzuführen, um geeignete Managements für Patienten zu schaffen, die von zugrunde liegenden Krankheiten und/oder Bedingungen betroffen sind, die potenzielle Risikofaktoren für die Prognose von Patienten darstellen, bei denen SARS-CoV-2 diagnostiziert wurde, um die von innen ausgehenden Komplikationen zu mildern (RENU; PRASANNA; GOPALAKRISHNAN, 2020; YANG et al., 2020).

\section{ZIELE}

Analyse der Verteilung der wichtigsten bereits bestehenden Risikofaktoren bei Patienten, bei denen COVID-19 im Bundesstaat Amapá, Amazônia, Brasilien diagnostiziert wurde.

\section{METHODIK}

Diese Studie zielte auf eine quantitative und beschreibende Analyse der Anzahl der von COVID-19 betroffenen Patienten in Amapá ab, die Risikofaktoren für die Krankheit bezeugen, und dazu wurde am 22. Mai 2020 eine Konsultation im Coronavirus Amapá Panel (painel.corona.ap.gov.br) durchgeführt.

Auf der Hauptseite des Coronavirus Amapá-Panels wurde auf "Download CSV" geklickt, um die wichtigsten Daten von covid-19 Fällen im Bundesstaat Amapá a zu erhalten. Dann, in den "Komorbiditäten der bestätigten Patienten" der gleichen Seite, wurde die Option "Inspect" in der oberen rechten Ecke ausgewählt, um eine Liste der Komorbiditäten und ihre numerische Beziehung zu generieren. Derselbe Prozess wurde in den "Komorbiditäten sterbender Patienten" wiederholt. Anschließend wurde für jede der erhaltenen Listen die Option "Fromatted CSV" im Feld "CSV herunterladen" ausgewählt, um die Dateien zu erhalten und in Microsoft Excel zu verwenden. 
Innerhalb dieser Dateien wurden einige Komorbiditäten und Risikosituationen im selben Satz verbunden, um die Ergebnisse zu standardisieren. Zu sein: Sie sind gruppiert -in chronischen Herzerkrankungen - chronische Herzerkrankungen, chronische Herzerkrankungen, chronische Herzerkrankungen, chronische Herzerkrankungen, chronische Herzerkrankungen, Herz-Kreislauf-Erkrankungen, Herzerkrankungen, kardiopathische Erkrankungen, chronische Herzerkrankungen, Herzerkrankungen; sind auch in - Chronische Atemwegserkrankungen - chronische Atemwegserkrankungen, chronische Atemwegserkrankungen, chronische Atemwegserkrankungen, chronische dekompensierte chronische Atemwegserkrankungen; Sie sind immer noch in - Hypertonie- Hypertonie, HAS, hypertensiv; wieder sind in - Herzerkrankungen und Bluthochdruck - Chronische Herzerkrankungen (HAS), chronische Herzerkrankungen - HAS, Herz-KreislaufErkrankungen einschließlich Bluthochdruck, chronische Herzerkrankungen HAS, Herzerkrankungen (HAS), chronische Herzerkrankungen - HAS; sowie in Immunsuppression- Immunsuppression, Chromosomenkrankheitträger oder FRZustand; Wieder sind in - Herzerkrankungen und Diabetes - chronische Herzerkrankungen und Diabetes, Herz und Diabetiker; So sind sie in HochrisikoSchwangerschaft - Hochrisiko schwangere Frau, Hochrisiko schwangere Frau; Endlich sind in - Advanced Stage Chronische Nierenerkrankungen - Fortgeschrittene Sendezeit chronische Nierenerkrankungen, Advanced-Stage Chronische Nierenerkrankung, Fortgeschrittene-Stadium chronische Nierenerkrankung.

In der Tabelle "Komorbiditäten von Patienten im Tod" wurde die Zahl der fälle, die als "Keine Informationen" registriert wurden, aufgrund der Unschlüssigkeit dieses Cutoffs nicht berücksichtigt. Die anderen Daten wurden wie oben beschrieben ausgewählt und gruppiert.

In der vorliegenden Studie wurden sekundäre Daten verwendet, die im Bereich des öffentlichen Zugangs verfügbar sind, und ethische Aspekte auf der Grundlage der Resolution 466/12 berücksichtigt. 


\section{ERGEBNISSE}

Von den 28.927 bestätigten Fällen von COVID-19 in Amapá, die bis zum Tag der Sammlung gemeldet wurden, hatten etwa 1,31\% der Patienten (378 Fälle) einen Risikofaktor, der sie anfällig für Komplikationen aufgrund der Beteiligung von SARSCoV-2 machen konnte, wie in Abbildung 1 dargestellt.

Abbildung 1- Vorhandensein von Risikofaktoren bei Patienten, bei denen COVID-19 in Amapa bis zum 22. Mai 2020 diagnostiziert wurde.

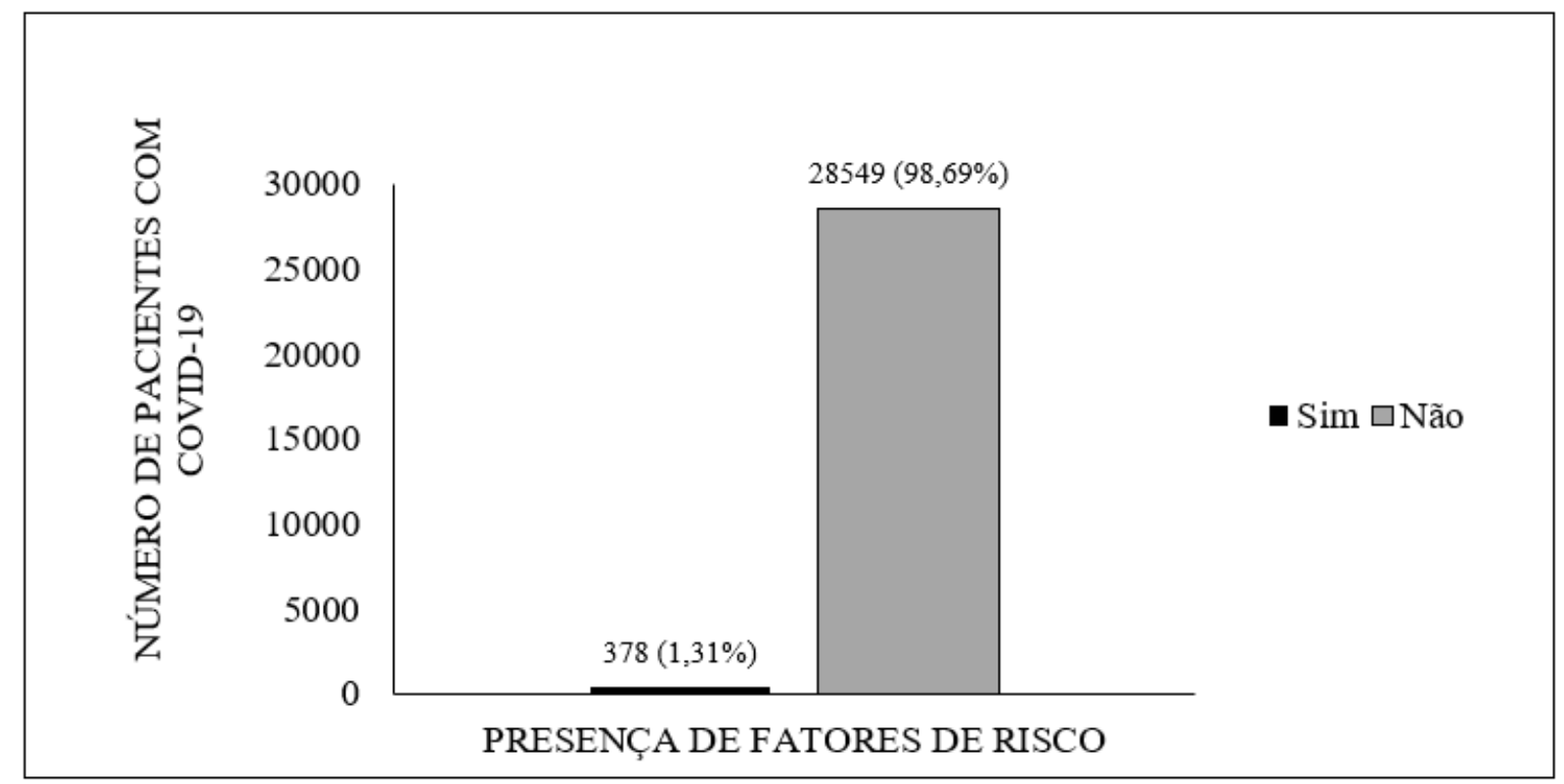

Quelle: Coronavirus Amapá-Panel.

Bei Patienten mit Risikofaktoren hatten 5,02\% von ihnen (19 Fälle) mehr als einen. Zu den Assoziationen gehören Herzerkrankungen und Bluthochdruck (3,44 \%), Herzerkrankungen und Diabetes (1,06\%), Angioplastie und koronare Atheromtose $(0,26 \%)$ chronischenierenund Diabetes $(0,26 \%)$.

$\mathrm{Zu}$ den wichtigsten Risikofaktoren, die allein von betroffenen Patienten berichtet wurden, wurde festgestellt, dass die Mehrheit Diabetes 29,89\% (112 Fälle) hatte, gefolgt von chronischen Herzerkrankungen mit 28,84\% (109 Fälle). 
Es wird bemerkt, dass Krankheiten der kardiologischen Sphäre in einer mehrheitsgemäßen Weise vorhanden sind, wenn man bedenkt, dass insgesamt 173 Patienten von Krankheiten dieser Klasse betroffen sind, wobei Personen mit mehreren Risikofaktoren und Personen nur mit Herzerkrankungen hinzugefügt werden.

Tabelle 1- Verteilung der Risikofaktoren bei Patienten, bei denen COVID-19 in Amapá diagnostiziert wurde.

\begin{tabular}{|c|c|c|}
\hline Risikofaktoren & $\begin{array}{l}\text { Anzahl der } \\
\text { Berichte }\end{array}$ & $\begin{array}{c}\text { Prozentsatz } \\
\qquad(\%)\end{array}$ \\
\hline Diabetes & 113 & $29,89 \%$ \\
\hline Chronische Herzerkrankung & 109 & $28,84 \%$ \\
\hline Chronische Atemwegserkrankungen & 71 & $18,78 \%$ \\
\hline Bluthochdruck & 46 & $12,17 \%$ \\
\hline Herzerkrankungen und Bluthochdruck & 13 & $3,44 \%$ \\
\hline Immunosuppression & 11 & $2,91 \%$ \\
\hline Herzerkrankungen und Diabetes & 4 & $1,06 \%$ \\
\hline Hochrisikoschwangerschaft & 4 & $1,06 \%$ \\
\hline $\begin{array}{l}\text { Fortgeschrittenes Stadium Chronischer } \\
\text { Chronischer Erkrankungen }\end{array}$ & 3 & $0,79 \%$ \\
\hline Angioplastie und koronare Atheromatose & 1 & $0,26 \%$ \\
\hline Chronische Herzerkrankungen und Diabetes & 1 & $0,26 \%$ \\
\hline Leberzirrhose & 1 & $0,26 \%$ \\
\hline Depression & 1 & $0,26 \%$ \\
\hline gesamt & 378 & $100,00 \%$ \\
\hline
\end{tabular}

Quelle: Coronavirus Amapá-Panel.

Insgesamt wurden zum Zeitpunkt der Studie 420 Todesfälle registriert. Von dieser quantitativen, Es wurde festgestellt, dass 318 Patienten hatten eine Geschichte der Komorbidität oder prädisponierenden Zustand zu Komplikationen. 
Abbildung 2 - Vorhandensein von Risikofaktoren bei Patienten, die bis zum 22. Mai 2020 mit COVID-19 in Amapá starben.

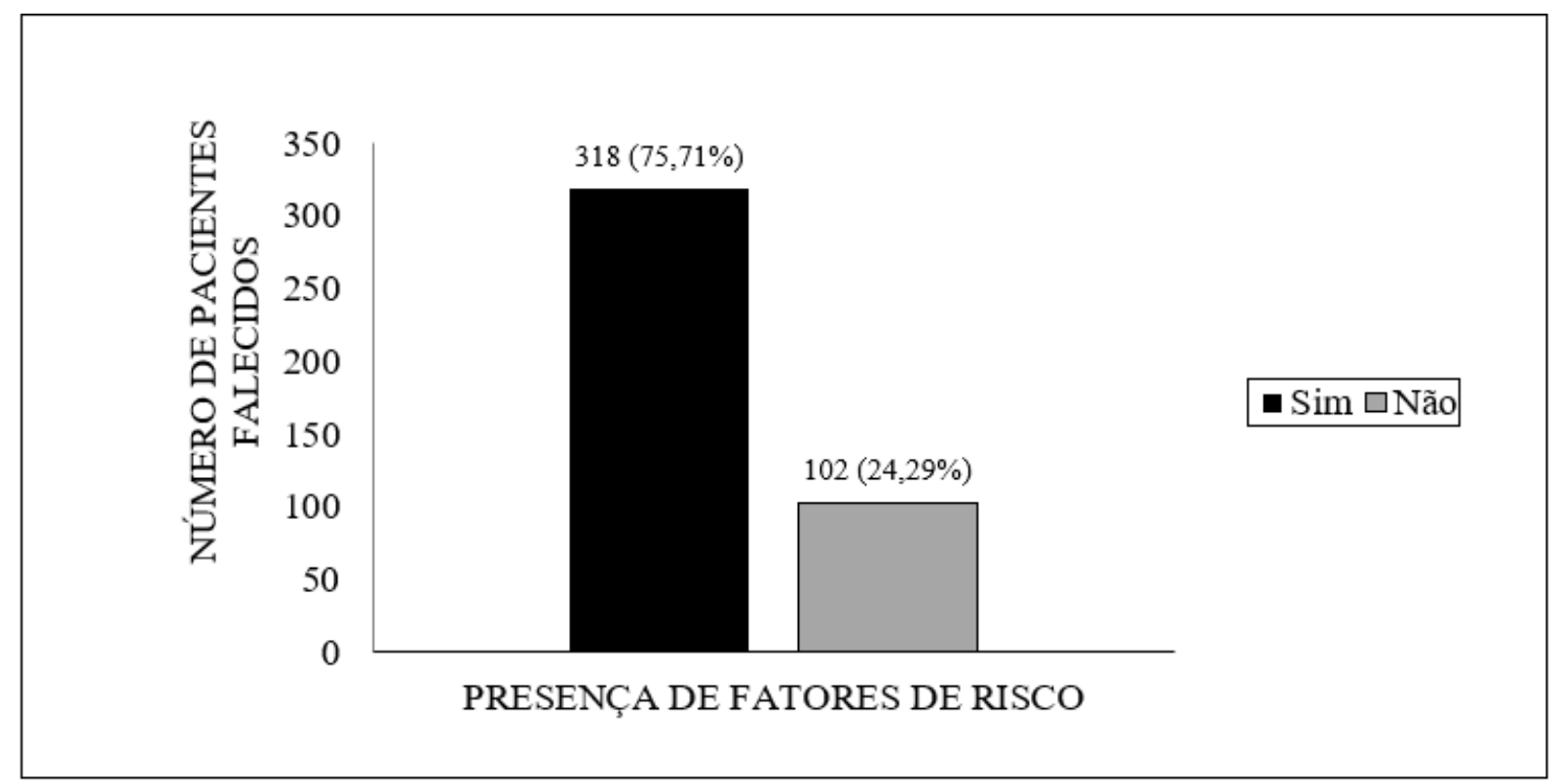

Quelle: Coronavirus Amapá-Panel.

Hinsichtlich der Risikofaktoren bei Patienten, die starben, war der Hauptdatensatz Bluthochdruck, der bei 38,68\% der Patienten (123 Fälle) vorhanden war. Es wurde auch das Vorhandensein von anderen erschwerenden Faktoren festgestellt, die zuvor nicht bei Personen mit bestätigter Infektion beschrieben wurden. Sie sind Fettleibigkeit, zerebrovaskuläre Erkrankungen, Neoplasie, Rauchen, Alzheimer, Bauchspeicheldrüsenerkrankungen, Dengue, Alkoholkonsum und Puerperalperiode. 
Tabelle 2 - Verteilung der Risikofaktoren, die bei Patienten erfasst wurden, die mit COVID-19 in Amapá starben.

\begin{tabular}{|l|c|c|}
\hline Komorbidität & Anzahl der Todesfälle & Prozentsatz (\%) \\
\hline Bluthochdruck & 123 & $38,68 \%$ \\
\hline Diabetes & 93 & $29,25 \%$ \\
\hline Krankheiten der Haut & 27 & $8,49 \%$ \\
\hline Herzen & 19 & $5,97 \%$ \\
\hline Fettleibigkeit & 19 & $5,97 \%$ \\
\hline Atemwegserkrankungen & 15 & $4,72 \%$ \\
\hline Zerebrovaskuläre Erkrankungen & 8 & $2,52 \%$ \\
\hline Immunsuppressive Erkrankungen & 3 & $0,94 \%$ \\
\hline Tumoren & 3 & $0,94 \%$ \\
\hline Rauchen & 2 & $0,63 \%$ \\
\hline Alzheimer & 1 & $0,31 \%$ \\
\hline Pankreas-Krankheit & 1 & $0,31 \%$ \\
\hline Lebererkrankung & 1 & $0,31 \%$ \\
\hline Dengue-Fieber & 1 & $0,31 \%$ \\
\hline Etilist & 1 & $0,31 \%$ \\
\hline Puerpera & 18 & $0,31 \%$ \\
\hline gesamt & $100,00 \%$ \\
\hline
\end{tabular}

Fonte: Painel Coronavírus Amapá.

\section{DISKUSSION}

Die Bundesstaaten im Amazonasgebiet Brasiliens weisen im Vergleich zu den anderen erhebliche sozioökonomische Unterschiede auf, eine Situation, die möglicherweise die größten Auswirkungen auf das beobachtete Gesundheitssystem widerspiegelt. Im Bundesstaat Amapá hat sich COVID-19 nach der Meldung des ersten Falls am 25. März 2020 massiv ausgebreitet, ebenso in anderen föderativen Einheiten (DIAS et al., 2020; MENDONÇA et al., 2020). 
Die Zahl der Patienten mit COVID-19, die Risikofaktoren für die Krankheit in Amapá a haben, im Vergleich zur Gesamtzahl der infizierten Patienten, wird als gering angesehen. In der Literatur herrscht Einigkeit darüber, dass Komorbiditäten und Situationen der immunologischen Verletzlichkeit als erschwerende Faktoren für den ZUSTAND COVID-19 angesehen werden, und diese Tatsache könnte Personen in den Risikogruppen veranlasst haben, ihre Pflege zu verdoppeln (COSTA et al., 2020b; RODRÍGUEZ-COLA et al., 2020).

In einer Studie von Wang et al. (2020) wurde berichtet, dass von 36 Patienten, die auf der Intensivstation (UTI) betreut werden mussten, 26 (72,2\%) komorbiditäten, eine Feststellung, die darauf hindeutet, dass eine Geschichte von chronischen Krankheiten kann zur Entwicklung von Komplikationen führen. Silva et al. (2020), in einer klinischen und epidemiologischen Charakterisierungsstudie mit 1560 Patienten mit COVID-19 aus der Stadt Macapá-AP diagnostiziert, fand heraus, dass 102 Patienten (6,54\%) Komorbiditäten.

Die wichtigsten Komorbiditäten, die allein bei den Patienten in der vorliegenden Studie festgestellt wurden, waren Diabetes, chronische Herzkrankheiten, chronische Atemwegserkrankungen und Bluthochdruck. Eine Meta-Analyse mit insgesamt 1576 Teilnehmern von Yang et al. (2020) hebt auch Bluthochdruck (21,1\%), Diabetes $(9,7 \%)$, Herz-Kreislauf-Erkrankungen (8,4\%) hervor. und Erkrankungen der Atemwege $(1,5 \%)$ als die häufigsten Komorbiditäten. Silva et al. (2020) ergab, dass von den 102 Patienten mit Komorbiditäten, die mit COVID-19 diagnostiziert wurden, chronische Herz-Kreislauf-Erkrankungen (38,33\%), Diabetes (24,16\%) und chronische Atemwegserkrankungen (13,33\%) waren die am weitesten verbreiteten Risikofaktoren. Diese Bedingungen sind mit einem hohen Risiko für schwerwiegende Manifestationen von COVID-19 (GOMES; GOMEZ, 2020; MARTINS-CHAVES), was zum Teil durch die erhöhte Expression des Angiotensin-Wandler-Enzyms 2 (ECA2) in Alveolar-, Herz-, $\beta$ Pankreas- und vaskulären Endothel-Epithelzellen erklärt wird. ECA2 ermöglicht das Eindringen des Virus in die Zellen des Körpers, eine Tatsache, die das Versagen der entsprechenden Organe während der Infektionskrankheit 
bestätigt (ANGHEBEM; REGO; PICHETH, 2020; ASKIN; TANRIVERDI; ASKIN, 2020).

Einige Patienten hatten auch andere Komorbiditäten und Risikofaktoren, einschließlich Immunsuppression, Hochrisikoschwangerschaft, chronische Nierenerkrankungen, Angioplastie mit koronarer Atheromtose, Leberzirrhose und Depression.

Die Daten der vorliegenden Studie zeigen eine Letalität bei Patienten mit immunsuppressiven Erkrankungen von 27,27\%. Es wird angenommen, dass je mehr die Immunität von Patienten mit COVID-19 beeinträchtigt, desto schlechter die Prognose der Krankheit, ein Grund, der immunsuppressive Patienten als Gruppen mit Risiko für die Krankheit rahmen (GOMES; GOMEZ, 2020; MARTINS-CHAVES). Es gibt jedoch einen Bericht in der Literatur, dass die meisten der immunsuppressiven Patienten, die bewertet wurden, mit einer guten Prognose folgten. Eine Studie von Hrusak et al. (2020) bei pädiatrischen Patienten, die sich einer Krebsbehandlung unterziehen, zeigte sich, dass von 9 Kindern, bei denen COVID-19 diagnostiziert wurde, 8 mit leichten Symptomen folgten oder sogar asymptomatisch waren. Außerdem, Bussalino et al. (2020) erklären, dass die Aufrechterhaltung der Immunsuppression den Verlauf von COVID-19 für den Patienten mild machen kann, indem der für die Krankheit charakteristische Zytokinsturm reduziert wird.

In Bezug auf die Schwangerschaft wurden 4 Fälle von Hochrisikoschwangerschaft im Staat gemeldet. Mütterliche Risiken werden vermutet, weil systemische und lokale immunologische Veränderungen im Körper der Mutter ausgelöst werden, die von einem entzündungshemmenden Zustand bis zu einem entzündungshemmenden Zustand reichen (LIU et al., 2020). Bei Schwangeren mit COVID-19 wurde eine Metaanalyse von Trippela et al. (2020) mit 275 schwangeren Frauen, die von der Krankheit betroffen waren, zeigte sich, dass 269 ihre Symptomatologie beschrieben, und $91,82 \%$ hatten Symptome, einschließlich Fieber und Husten als die häufigsten, und $8,18 \%$ waren asymptomatisch. Die meisten Patienten zeigen leichte oder asymptomatische Symptome, mit wenigen Berichten über die Entwicklung von Komplikationen (CAPARROS-GONZALEZ, 2020; TRIPPELA et al., 2020). 
In Bezug auf chronische koronare Erkrankungen wurden nur 3 bestätigte Fälle an das epidemiologische Überwachungssystem des Amapá-Coronavirus gemeldet. Allerdings wurden 27 Fälle von Todesfällen von Patienten, die zuvor Komorbiditäten dieser Klasse von Krankheiten hatten, in das System eingeführt, was auf eine mögliche Untererfassung von Komorbiditäten hindeutet. In einer prospektiven Kohortenstudie von Cheng et al. (2020) bei 701 Personen, die von COVID-19 betroffen waren, wurde festgestellt, dass 26,7 \% der befragten Personen Anhäamature und 43,9\% Proteinurie hatten. Diese klinischen Befunde deuten auf Laborprobleme hin (MOITINHO et al., 2020). DA SARS-CoV-2 direkt auf den ECA2-Empfänger wirkt (ASKIN; TANRIVERDI; ASKIN, 2020) und die bereits eine Überexpression dieses Enzyms in den proximalen röhrenförmigen Zellen von Patienten mit chronischen Oralerkrankungen nachgewiesen wurde (ANA et al., 2020), wird angenommen, dass die Geschichte der Lebenskrankheiten das Bild von COVID-19 (RENU; PRASANNA; GOPALAKRISHNAN, 2020). Darüber hinaus wurde es mit der Vorexistenz von Nierenerkrankungen mit der Beeinträchtigung der Funktionalität des Immunsystems des Patienten in Verbindung gebracht, ein Prozess, der durch den entzündlichen Prozess während der Nephropathie erklärt werden könnte. Dies fördert die Vorstellung, dass diese Krankheiten Risikofaktoren für COVID-19 (OYELADE; ALQAHTANI; CANCIANI, 2020).

In Bezug auf die Beziehung zwischen Angioplastie und koronarer Atheromtose mit COVID-19 wurden keine Berichte in der Literatur gefunden, die den Zusammenhang zwischen diesen Risikofaktoren und einer Virusinfektion unterstützen könnten.

Von den gemeldeten Fällen von Lebererkrankungen im Bundesstaat Amapá wurde nur 1 Fall von Leberzirrhose gemeldet. Der Tod eines Patienten mit einer Lebervorerkrankung wurde ebenfalls erfasst, jedoch kann nicht gesagt werden, dass es sich um denselben Patienten handelt, da die Vorerkrankung nicht angegeben wurde. Die Literatur zeigt, dass die Infektion durch SARS-CoV-2 auch die Leber betrifft (AGHAGOLI et al., 2020) und das Vorliegen von Lebererkrankungen als bestimmender Faktor für die Schwere des COVID-19-Bildes gilt (RENU; PRASANNA; GOPALAKRISHNAN , 2020). Eine von Oyelade, Alqahtani und Canciani (2020) 
durchgeführte Metaanalyse mit 5595 Patienten zeigte eine Prävalenz von Lebererkrankungen bei Patienten mit COVID-19 von $3 \%$, von denen $57,33 \%$ einen Schweregrad hatten. Dieselbe Studie ergab eine Sterblichkeitsrate von 17,65 \%. Trotz der Schwere wurde in der Literatur kein Zusammenhang zwischen einer vorbestehenden Lebererkrankung und dem Ergebnis von COVID-19 gefunden.

Im einzigen Fall von Depressionen, die im Staat registriert sind, gibt es eine Sorge der wissenschaftlichen Gemeinschaft über die Möglichkeit von neuropsychischen Erkrankungen, die auch ein Risiko für den ZUSTAND COVID-19 darstellen. Psychische Erkrankungen sowie der Zustand psychischer Not beeinflussen direkt die Funktion des Immunsystems eines Individuums, das sie anfällig für Virusinfektionen der Atemwege sein kann, was einen möglichen Zusammenhang zwischen dem emotionalen Zustand des Patienten und dem Grad der Beteiligung von SARS-CoV-2 belegt (RAJKUMAR, 2020). Die durch das Virus erzeugten Wirkungen auf die betreffenden Patienten sowie die vom Organismus erzeugte immunologische Reaktion sind in der Literatur jedoch nicht gut aufgeklärt (TROYER; KOHN; HONG, 2020). Bei Patienten mit einer Vorgeschichte von psychischer Schwäche sollte der Erkrankung größere Aufmerksamkeit geschenkt werden, da sie inmitten des installierten Pandemieszenarios und einer möglichen Diagnose von COVID-19 (YAO; CHEN; XU, 2020).

Die daten, die aus dem Amap-Coronavirus-Panel gesammelt wurden, zeigen, dass 76 $\%$ der Patienten, die starben, Komorbiditäten oder bereits bestehende erschwerende Faktoren hatten. Eine epidemiologische Untersuchung von Almeida et al. (2020) im Bundesstaat Maranhéo stellte fest, dass von den 100 Patienten, bei denen COVID-19 diagnostiziert wurde und die starben, 78 \% frühere Komorbiditäten hatten, wobei sie ein ähnliches Muster wie in Amapá a gt;

Es wird beobachtet, dass Bluthochdruck der amhäufigste Risikofaktor bei amapaense Patienten ist, die starben. Es ist jedoch nicht möglich, die Tödlichkeitsrate bei hypertensiven Patienten zu bestimmen, da die Anzahl der bestätigten Fälle der Krankheit niedriger ist als die des Todes gemeldeten. Dennoch zeigt die Literatur, dass 
diese Krankheit einer der haupterschwerenden Faktoren für die COVID-19Erkrankung ist, zusammen mit anderen Herz-Kreislauf-Erkrankungen (AGHAGOLI et al., 2020; ALMEIDA et al., 2020; FERRARI, 2020). Der pathophysiologische Mechanismus, der vorgeschlagen wurde, um diese Beziehung zu erklären, beinhaltet das Reinthin-System. Aufgrund der höheren Bindung von SARS-CoV-2 an ECA2 wird die Konzentration dieses Enzyms im Wirt reduziert, was zu hohen Angiotensin-IISpiegeln und vermindertem Angiotensin 1-7 führt, Wirkungen, die Vasokonstriktion und Natriumretention erzeugen und den hypertensiven Zustand verschlechtern (TADIC et al., 2020).

In Bezug auf die Beziehung zwischen Diabetes und COVID-19 ist es möglich, die klinischen Bedingungen des Patienten zu verschlechtern, wenn man bedenkt, dass SARS-COV-2 erhöhte Blutzuckerspiegel bei Patienten mit Typ-2-Diabetes fördert (BORNSTEIN et al., 2020). Eine Verschlechterung des Zustands von Diabetikern kann damit zusammenhängen, dass diese Komorbidität die natürliche Immunität des Körpers verringert (COSTA et al., 2020b; MA; HOLT, 2020). In Bezug auf die gemeldeten Fälle dieser Krankheit in Amapá, ist es auffällig, dass die Anzahl der bestätigten Patienten (112) ist quantitativ nah an denen, die starben (93), so dass die Idee, dass Diabetes kann die covid-19 Zustand zu verschlimmern, bis zu dem Punkt, dass der Patient stirbt (COSTA et al., 2020b; RONCON et al., 2020).

Ein weiteres wichtiges Merkmal der Diabetes- und SARS-COV-2-Beziehung sind die Mechanismen der Komplikationen, die sich aus der Vereinigung dieser beiden Faktoren ergeben, da Personen, die Diabetes haben und mit einem Virus infiziert sind, einem stärkeren Risiko für Komplikationen ausgesetzt sind (LACOBELLIS, 2020). Eine Hypothese über Diabetes, der negativ wirkt, zusammen mit dem Virus, steht im Zusammenhang mit ECA2, da es in mehreren Geweben vorkommt und als Tor zu Infektionserregern auf der Zelloberfläche dient (AGHAGOLI et al., 2020; MA; HOLT, 2020). Darüber hinaus entwickeln Patienten, die Angiotensin-Suppressing Enzyme Inhibitoren (ACEI) verwenden, eher Komplikationen aufgrund einer höheren ECA2Produktion, was die Wirkung von SARS-CoV-2 begünstigt (FANG; KARAKIULAKIS; ROTH, 2020; FERRARI, 2020; MA; HOLT, 2020). 
Herzerkrankungen wiederum sind Bedingungen, die mit schlechteren Prognosen und hohen Tödlichkeitsraten verbunden sind (AGHAGOLI et al,4020). Eine Metaanalyse von Huang, Wang und Li (2020) ergab, dass von den 41 untersuchten Patienten mit COVID-19 6 (15\%) Erkrankungen der Herzsphäre hatte. Tatsächlich gibt es eine beträchtliche Anzahl von Patienten mit Herzerkrankungen, die in Amapá (19) starben, was eine Tödliche Rate von 15,07 \% erzeugte. Die Literatur assoziiert diese Schwere mit der Anwesenheit von ECA2-Rezeptoren im Herzen, die stark im Organ exprimiert werden, was zur Entwicklung von akuten Myokardverletzungen und chronischen HerzKreislauf-Schäden führen kann (AGHAGOLI et al., 2020). Darüber hinaus kann der bei den meisten Patienten mit Herzerkrankungen beobachtete höhere Alters- und Immunseneszenzeffekt mit der Prognose der COVID-19-Erkrankung (DRIGGIN et al., 2020).

Eine beträchtliche Anzahl von Patienten mit Atemwegserkrankungen wurde ebenfalls beobachtet, was einer Tödlichkeitsrate von 21,12\% unter Berücksichtigung der Anzahl der Patienten mit COVID-19 entspricht, die bereits bestehende Komorbiditäten berichteten. Die Vorexistenz von Atemwegserkrankungen ist mit schweren Erkrankungen von COVID-19 (MARTINS-CHAVE; GOMES; GOMEZ, 2020; YANG et al., 2020). Es ist bekannt, dass das Immunsystem auf eine Infektion durch SARS-CoV2 mit einem entzündlichen Prozess reagiert, der durch die Wirkung von Immunzellen, Gewebenekrose und lokaler Hyperplasie gekennzeichnet ist, Effekte, die den Prozess des Gasaustauschs der Lunge beeinträchtigen und ein Bild von schwerer Lungenentzündung erzeugen können (GIMENEZ et al., 2020), was rechtfertigen kann, warum Atemwegserkrankungen ein Risikofaktor für COVID-19 sind.

Auch in den Informationen, die vom Coronavirus Amapá-Panel zur Verfügung gestellt werden, wird beobachtet, dass einige Risikofaktoren für COVID-19 bei Patienten, die starben, nicht aus der Sitzung von Risikofaktoren von Patienten berichtet wurden, die mit der Krankheit diagnostiziert wurden, wie Fettleibigkeit, zerebrovaskuläre Erkrankungen, Neoplasmen, Rauchen, Alzheimer-Krankheit, Bauchspeicheldrüsenerkrankung, Dengue, Alkoholism und Puerperalzeit. Dies 
verhindert eine zuverlässige Analyse des epidemiologischen Szenarios des Ortes. Einige Aspekte dieser Bedingungen wurden jedoch in der Literatur erörtert.

Über Adipositas, Es ist seit langem bekannt, dass es direkt mit schlechten Prognosen und zeitaufwändige Erholung in Fällen von Virusinfektionen verbunden ist. Dieser Befund wird auf den konstanten entzündlichen Zustand zurückzuführen, der für adipöse Personen charakteristisch ist, unterstützt durch hohe Konzentrationen von entzündlichen Medikamenten, was zur Beeinträchtigung der immunologischen Reaktion dieser Patienten und folglich zur Abnahme der Fähigkeit zur Bekämpfung von Infektionserregern beiträgt (COSTA et al., 2020b; HUSSAIN et al., 2020; KORAKAS et al., 2020). Im Zusammenhang mit COVID-19 können andere Faktoren im Zusammenhang mit Adipositas für die höheren Chancen verantwortlich sein, das klinische Bild zu verschlechtern, das eine mangelhafte Atemmechanik, einen niedrigen Gasaustausch in der Lunge, eine erhöhte Atemwegsresistenz, eine verminderte Lungenstärke und ein geringeres Lungenvolumen umfasst (STEFAN et al., 2020). Eine Metaanalyse von Hussain et al. (2020) zeigte, dass von den 2.451 Patienten mit Body Mass Index (IMC) größer als $25 \mathrm{~kg} / \mathrm{m} 2531$ starben, was einer Tödlichkeitsrate von $21,66 \%$ entspricht. In derselben Studie wurden auch Patienten mit einem BMI von weniger als $25 \mathrm{~kg} / \mathrm{m}^{2}$ bewertet,was 24.506 betrug. Davon starben 1701, eine Tödliche Rate von 6,94\%. Der Vergleich dieser Werte zeigt, dass Übergewicht und Adipositas die Prognose des Patienten beeinflussen können.

Zerebrovaskuläre Erkrankungen wurden in schwereren Fällen von COVID-19 (WANG et al., 2020). Der Mechanismus hinter der Zunahme der Schwere für diese Klasse von Patienten ist immer noch ungewiss, obwohl es bereits vermutet wird, dass es ähnlich wie bei Herz-Kreislauf-Erkrankungen ist (PRANATA et al., 2020).

In Bezug auf die Beziehung zwischen Neoplasmen und COVID-19, Es wird angenommen, dass Krebspatienten anfällig für die Entwicklung von Komplikationen aufgrund der Schwächung ihrer immunologischen Aktivität sind (ASOKAN; RABADIA; YANG, 2020; AL-QUTEIMAT; AMER, 2020). In der Tat, eine retrospektive Studie von Zhang et al. (2020) zeigte, dass von 28 untersuchten Krebspatienten 8 starben 
(Sterblichkeitsrate von 28,6\%), was auf eine schlechte Prognose für Patienten mit Neoplasmen vor der Existenz hindeutet.

Die Geschichte des Rauchens wiederum wird als wichtiger Risikofaktor für die Entwicklung von schweren Erkrankungen der Krankheit spekuliert, da Rauchen mit eingeschränkter Lungengesundheit verbunden ist (VARDAVAS; NIKITARA, 2020). Obwohl nur ein Fall von Rauchen bei Patienten berichtet wurde, die in unserer epidemiologischen Analyse starben, gibt es Studien, die ein erhöhtes Schwererisiko bei Patienten mit einer Vorgeschichte des Rauchens zeigen. Alqahtani et al. (2020), in einer Meta-Analyse mit 139 aktuellen Rauchern und 28 ehemaligen Rauchern mit COVID-19 diagnostiziert, festgestellt, dass 31 aktuelle Raucher (22,30\%) und 13 ehemalige Raucher (46\%) verschlechterte sich ihrer klinischen Bedingungen. In der gleichen Studie starben 5 der 13 Raucher, die Komplikationen entwickelten (Sterblichkeitsrate von 38,5\%). Diese Beobachtungen bestätigen nur, dass Personen, die bereits geraucht oder geraucht haben, die Praxis inmitten der Pandemie vermeiden und immer vorbeugende Maßnahmen ergreifen sollten.

Was die Alzheimer-Krankheit betrifft, den Zusammenhang zwischen fortgeschrittenem Alter und Demenz, bemerkenswerte Merkmale der Krankheit sowie die mögliche Beeinträchtigung der neurokognitiven Entwicklung dieser Personen während der sozialen Isolation in der Pandemie, ermutigen die wissenschaftliche Gemeinschaft, die Vorexistenz von Alzheimer als Risikofaktor für COVID-19 zu bewerten (BRASIL, 2020b; FERINI-STRAMBI; SALSONE, 2020). Im Falle der Sterblichkeit, Bianchetti et al. (2020) ergab, dass von 82 Patienten, bei denen COVID-19 diagnostiziert wurde und an Demenz litt, 51 (62,2\%) starb, eine Rate höher als die von 26,2\% bei Patienten, die keine Demenz hatten, was darauf hinweist, dass nicht nur Alzheimer, sondern auch andere Demenzerkrankungen mit einer schweren Kovid-19-Erkrankung in Verbindung gebracht werden können.

Der gemeldete Fall eines verstorbenen Etilisten deutet auf Gewohnheit als möglichen Risikofaktor für die Krankheit hin. Dieser Zusammenhang wird durch die Vorkenntnisse unterstützt, dass Alkoholmissbrauch die Leberfunktion beeinträchtigen kann 
(EHRMANN; URBAN; DVORAN, 2019), was den Patienten erschrecken würde, Komplikationen und damit ein schwereres Bild von COVID-19 zu entwickeln.

Die Beziehung zwischen Puerperium und COVID-19 ist im Vergleich zur Schwangerschaft nicht so gut etabliert (AN et al., 2020). Tutiya et al. (2020) berichtete über zwei Fälle von pulmonaler Mikrothrombenbildung bei Puerperalfrauen, bei denen COVID-19 diagnostiziert wurde. Außerdem, An et al. (2020) berichtete von zwei Fällen von Puerperalfrauen, die Atemnot und eine Verschlechterung des Zustandes nach der Entbindung zeigten. Diese Beobachtungen verstärken die Notwendigkeit, den Zustand der Patienten nach der Entbindung zu überwachen, bis es eine Stabilisierung gibt.

Dengue- und Bauchspeicheldrüsenerkrankungen, die bei Todesfällen aufgrund von COVID-19 in Amapa gemeldet wurden, wurden jedoch in der Literatur keine Berichte über die Verbindung dieser Krankheiten und die Entwicklung von Komplikationen einer Virusinfektion gefunden, obwohl Krankheiten, die die Immunität des Patienten beeinträchtigen, dazu neigen, die Wahrscheinlichkeit einer Verschlechterung der vorbestehenden Erkrankungen zu erhöhen (MARTINS-CHAVES; GOMES; GOMEZ, 2020).

\section{SCHLUSSFOLGERUNG}

Die Vorexistenz von Komorbiditäten oder anderen Bedingungen, die die Immunantwort des Körpers beeinträchtigen, ist ein potenzieller erschwerender Faktor des infektiösen Zustands, der durch das neue Coronavirus erzeugt wird, was den Patienten zum Tod führen kann. Im Bundesstaat Amapá hatte ein großer Teil der Bevölkerung von Patienten, bei denen die Krankheit diagnostiziert wurde und die starben, eine Vorgeschichte von Risikofaktoren für COVID-19.

Die wichtigsten Komorbiditäten, die bei Patienten berichtet wurden, bei denen die Krankheit diagnostiziert wurde, waren Diabetes, chronische Herzerkrankungen, chronische Atemwegserkrankungen und Bluthochdruck. Die Komorbidität mit der höchsten Bilanz bei Todesfällen war Bluthochdruck. 
Die Meldung von Risikofaktoren in der Todestabelle, die nicht im Bild der diagnostizierten Patienten aufgezeichnet wurden, sowie die Unspezifität, die dem Zustand der Patienten innewohnt, die von den Gesundheitsmechanismen des Staates überwacht werden, machten eine detailliertere Analyse über den Grad der Komplikation und der Tödlichen Rate, die durch eine gegebene Komorbidität erzeugt wurden, wenn sie mit einer Coronavirus-Infektion in Verbindung gebracht wurde.

Für eine größere Vollständigkeit und Zuverlässigkeit der Ergebnisse einer Studie wie dieser ist es unerlässlich, dass ausreichend genaue und kohärente Daten gewonnen werden, damit der Durchgang der Patienten durch das Gesundheitsnetz analysiert und dann in die wissenschaftliche Forschung einbezogen werden kann, so dass die aktuelle Pandemie und ihre Auswirkungen in Amap zu verstehen und zu bewältigen sind.

\section{VERWEISE}

AGHAGOLI, G. et al. Cardiac involvement in COVID-19 patients: Risk factors, predictors, and complications: A review. J Card Sug., v. 35, p. 1302-1305, abr. 2020.

ALMEIDA, J. S. et al. Caracterização epidemiológica dos casos de covid-19 no maranhão: uma breve análise. Revista Prevenção de Infecção e Sáude, v. 6, mai. 2020.

AL-QUTEIMAT, O. M.; AMER, A. M. The Impact of the COVID-19 Pandemic on Cancer Patients. Am J Clin Oncol., abr. 2020. DOI: 10.1097/COC.0000000000000712.

ALQAHTANI, J. S. et al. Prevalence, Severity and Mortality associated with COPD and Smoking in patients with COVID-19: A Rapid Systematic Review and Meta-Analysis. PLoS One, v. 15, n. 5, e0233147, mai. 2020.

AN, P. et al. Postpartum exacerbation of antenatal COVID-19 pneumonia in 3 women. CMAJ, v. 192, n. 22, p. 603-606, jun. 2020. 
ANGHEBEM, M. I.; REGO, F. G. M.; PICHETH, G. COVID-19 e Diabetes: a relação entre duas pandemias distintas. Revista Brasileira de Análises Clínicas. DOI: 10.21877/2448-3877.20200001. 2020.

ASKIN, L.; TANRIVERDI, O.; ASKIN, H. S. The Effect of Coronavirus Disease 2019 on Cardiovascular Diseases. Arq. Bras. Cardiol., São Paulo, v. 114, n. 5, p. 817-822, mai. 2020.

ASOKAN, I.; RABADIA, S. V.; YANG, E. H. The COVID-19 Pandemic and its Impact on the Cardio-Oncology Population. Curr Oncol Rep., v. 22, n. 6, mai. 2020.

BIANCHETTI, A. et al. Clinical presentation of COVID-19 in dementia patients. J Nutr Health Aging, mai., 2020. DOI: 10.1007/s12603-020-1389-1.

BORNSTEIN, S. R. et al. Practical recommendations for the management of diabetes in patients with COVID-19. The lancet Diabetes \& endocrinology, v. 9, n. 6, p. 546550, jun. 2020.

BRASIL. Plano de contingência DATASUS: Situação de crise provocada pelo novo coronavírus (covid-19). Versão 1.0, 2020a.

BRASIL. MINISTÉRIO DA SAÚDE. Alzheimer: o que é, causas, sintomas, tratamento, diagnóstico e prevenção. Disponível em:<https://saude.gov.br/saudede-a-z/alzheimer>. Acesso em: 31 jul. $2020 b$.

BRASIL. MINISTÉRIO DA SAÚDE. Painel de casos de doença pelo coronavírus 2019 (COVID-19) no Brasil pelo Ministério da Saúde. Versão 2.0. Disponível em:< https://covid.saude.gov.br/>. Acesso em: 10 de jul. de 2020c.

BUSSALINO, E. et al. Immunosuppressive therapy maintenance in a kidney transplant recipient SARS-CoV-2 pneumonia: a case report. Am J Transplant., abr. 2020. DOI: 10.1111/ajt.15920. 
CAPARRO-GONZALEZ, R. A. COVID-19 in PregnantWomen and Neonates: A Systematic Review of the Literature with Quality Assessment of the Studies. Rev Esp Salud Pública., v. 94, abr. 2020.

CHENG, $Y$ et al. Kidney disease is associated with in-hospital death of patients with COVID-19. Kidney Internacional, v. 97, n. 5, p. 829-838, mar. 2020.

CHINAZZI, M. et al. The effect of travel restrictions on the spread of the 2019 novel coronavirus (COVID-19) outbreak. Science, v. 368, n. 6489, p. 395-400, mar. 2020.

COSTA, I. B. S. S. et al. O Coração e a COVID-19: O que o Cardiologista Precisa Saber. Arq. Bras. Cardiol., v. 114, n. 5, p. 805-816, mai. 2020a.

COSTA, F. F. et al. Metabolic syndrome and COVID-19: An update on the associated comorbidities and proposed therapies. Diabetes \& Metabolic Syndrome: Clinical Research \& Reviews, v. 14, n. 5, p. 809-814, 2020 b.

DIAS, N. L. et al. Predição da propagação do SARS-CoV-2 no Estado do Amapá, Amazônia, Brasil, por modelagem matemática. Revista Científica Multidisciplinar Núcleo do Conhecimento, vol. 06, ano 05, ed. 05, p 73-95, mai., 2020.

DRIGGIN, E. et al. Cardiovascular Considerations for Patients, Health Care Workers, and Health Systems During the COVID-19 Pandemic. J Am Coll Cardiol., v. 75, n. 18, p. 2352-2371, mai. 2020.

ENGIN, A. B.; ENGIN, E. D.; ENGIN, A. Two important controversial risk factors in SARS-CoV-2 infection: obesity and smoking. Environmental Toxicology and Pharmacology, v. 78, mai. 2020. DOI: 10.1016/j.etap.2020.103411.

ENGSTROM, E. et al. Recomendações para a organização da Atenção Primária à Saúde no SUS no enfrentamento da Covid-19. Observatório Covid-19 Fiocruz, Nota técnica. p. 13, mai. 2020. 
EHRMANN, J.; URBAN, O.; DVORAN, P. Alcohol-related liver diseases. Cent Eur J Public Health, v. 27, p. 10-14, dez. 2019.

FAN, C. et al. ACE2 Expression in Kidney and Testis May Cause Kidney and Testis Damage After 2019-nCoV Infection. MedRxiv, fev. 2020. DOI: 10.1101/2020.02.12.20022418.

FANG, L.; KARAKIULAKIS, G.; ROTH, M. Are patients with hypertension and diabetes mellitus at increased risk for COVID-19 infection?. The Lancet. Respiratory Medicine, v. 8, n. 4, mai. 2020.

FERINI-STRAMBI, L.; SALSONE, M. COVID-19 and neurological disorders: are neurodegenerative or neuroimmunological diseases more vulnerable?. J Neurol., jul. 2020. DOI: 10.1007/s00415-020-10070-8.

FERRARI, F. COVID-19: Dados Atualizados e sua Relação Com o Sistema Cardiovascular. Arq. Bras. Cardiol., v. 114, n. 5, p. 823-826, mai. 2020. DOI: 10.36660/abc.20200215.

GAUTRET, P. et al. Hydroxychloroquine and azithromycin as a treatment of COVID19: results of an open-label non-randomized clinical trial. International journal of antimicrobial agents, mar. 2020. DOI: 10.1016/j.jjantimicag.2020.105949.

GIMENEZ, V. M. M. et al. Lungs as target of COVID-19 infection: Protective common molecular mechanisms of vitamin $\mathrm{D}$ and melatonin as a new potential synergistic treatment. Life Sci., v. 354, ago. 2020. DOI: 10.1016/j.lfs.2020.117808.

GUAN, W. et al. Clinical characteristics of coronavirus disease 2019 in China. New England journal of medicine, v. 382, n. 18, p. 1708-1720, fev. 2020.

HONG, H. et al. Clinical characteristics of novel coronavirus disease 2019 (COVID-19) in newborns, infants and children. Pediatrics and Neonatology, v. 61, n. 2, p. 131132, abr. 2020. 
HRUSAK, O. et al. Flash survey on severe acute respiratory syndrome coronavirus-2 infections in paediatric patients on anticancer treatment. European Journal of Cancer, v. 132, p. 11-16, abr. 2020.

HUANG, C.; WANG, Y.; LI, X. Características clínicas de pacientes infectados com o novo coronavírus de 2019 em Wuhan, China. Lanceta, v. 395, p. 497-506, jan. 2020.

HUSSAIN, A. Obesity and mortality of COVID-19. Meta-analysis. Obes Res Clin Pract., jul. 2020. DOI: 10.1016/j.orcp.2020.07.002.

KHALIQ, O. P.; PHOSWA, W. N. Is Pregnancy a Risk Factor of COVID-19?. European Journal of Obstetrics \& Gynecology and Reproductive Biology, 2020. DOI: 10.1016/j.ejogrb.2020.06.058.

IACOBELLIS, G. COVID-19 and diabetes: can DPP4 inhibition play a role?. Diabetes research and clinical practice, v. 162, abr. 2020. DOI: 10.1016/j.diabres.2020.108125.

LI, H. et al. Coronavirus disease 2019 (COVID-19): current status and future perspective. International journal of antimicrobial agents, v. 55, n. 5, mai. 2020.

LIU, H. et al. Why are pregnant women susceptible to COVID-19? An immunological Viewpoint. Journal of reproductive immunology, v. 139, jun. 2020.

LIMA, D. L. P. et al. COVID-19 no estado do Ceará, Brasil: comportamentos e crenças na chegada da pandemia. Ciênc. saúde coletiva, v. 25, n. 5, p. 1575-1586, mai. 2020.

LUCENA, T. M. C. et al. Mechanism of inflammatory response in associated comorbidities in COVID-19. Diabetes \& Metabolic Syndrome: Clinical Research \& Reviews, mai. 2020. DOI: 10.1016/j.dsx.2020.05.025.

MA, R. C. W.; HOLT, R. I. G. COVID-19 and diabetes. Diabetic Medicine, abr. 2020. DOI: $10.1111 / \mathrm{dme} .14300$. 
MARTINS-CHAVES, R. R.; GOMES, C. C.; GOMEZ, R. S. Immunocompromised patients and coronavirus disease 2019: a review and recommendations for dental health care. Braz. res oral., São Paulo, v. 34, e048, mai. 2020.

MENDONÇA, F. D. Região Norte do Brasil e a pandemia de COVID-19: análise socioeconômica e epidemiológica. Journal Health NPEPS, v. 5, n. 1, 2020.

MOITINHO, M. S. et al. Lesão renal aguda pelo vírus SARS-COV-2 em pacientes com COVID-19: revisão integrativa. Rev. Bras. Enferm., Brasília, v. 73, n. 2, jul. 2020.

OLIVEIRA, A. C.; LUCAS, T. C.; IQUIAPAZA, R. A. What has the COVID-19 pandemic taught us about adopting preventive measures?. Texto \& Contexto Enfermagem, Florianópolis, v. 29, mai. 2020. DOI: 10.1590/1980-265x-tce-2020-0106.

OLIVEIRA, W. K. et al. Como o Brasil pode deter a COVID-19. Epidemiol. Serv. Saude, Brasília, v. 9, n. 2, p. 1-8, abr. 2020.

OMS. ORGANIZAÇÃO MUDIAL DA SAÚDE. Painel da Doença de Coronavírus da OMS (COVID-19). Disponível em:< https://covid19.who.int/>. Acesso em: 10 de jul. de 2020.

OYELADE, T.; ALQAHTANI, J.; CANCIANI, G. Prognosis of COVID-19 in Patients with Liver and Kidney Diseases: An Early Systematic Review and Meta-Analysis. Trop. Med. Infect. Dis., v. 5, n. 80, mai. 2020.

PRANATA, R. et al. Impact of cerebrovascular and cardiovascular diseases on mortality and severity of COVID-19-systematic review, meta-analysis, and metaregression. J Stroke Cerebrovasc Dis., v. 29, n. 8,: mai. 2020.

PIRES, L. N.; CARVALHO, L.; XAVIER, L. L. COVID-19 e desigualdade: a distribuição dos fatores de risco no Brasil. Research Gate, abr. 2020. DOI: 10.13140/RG.2.2.27014.73282. 
RAJKUMAR, R. P. Ayurveda and COVID-19: Where psychoneuroimmunology and the meaning response meet. Brain, behavior and immunity, v. 87, p. 8-9, jul. 2020.

RENU, K.; PRASANNA, P. L.; GOPALAKRISHNAN, A. V. Coronaviruses pathogenesis, comorbidities and multi-organ damage - A review. Life sci., v. 255, mai. 2020. DOI: 10.1016/j.Ifs.2020.117839.

RODRÍGUEZ-COLA, M. et al. Clinical features of coronavirus disease 2019 (COVID19 ) in a cohort of patients with disability due to spinal cord injury. Spinal Cord Series and Cases, v. 6, n. 39, mai. 2020.

RONCON, L. et al. Diabetic patients with COVID-19 infection are at higher risk of ICU admission and poor short-term outcome. Journal of Clinical Virology, v. 127, jun. 2020. DOI: 10.1016 / j.jcv.2020.104354.

RUBIO-PÉREZ, I. et al. COVID-19: Conceptos clave para el cirujano. Cirugía Española, v. 98, n. 6, p. 310-319, jun./jul. 2020.

SIA, S. F. et al. Pathogenesis and transmission of SARS-CoV-2 in golden hamsters. Nature, mai. 2020. DOI: 10.1038/s41586-020-2342-5.

SILVA, A.W.C. et al. Caracterização clínica e epidemiologia de 1560 casos de COVID19 em Macapá/AP, extremo norte do Brasil. Research, Society and Development, v. 9, n.8, e150985499, jun. 2020.

STEFAN. N. et al. Obesity and impaired metabolic health in patients with COVID-19. Nat Rev Endocrinol., abr. 2020. DOI: 10.1038/s41574-020-0364-6.

TAY, M. Z. et al. The trinity of COVID-19: immunity, inflammation and intervention. Nat Rev Immunol, v. 20, p. 363-374, abr. 2020. DOI: 10.1038/s41577-020-0311-8.

TADIC, M. et al. COVID-19 and arterial hypertension: Hypothesis or evidence? J Clin Hypertens (Greenwich), jul. 2020. DOI:10.1111/jch.13925. 
TRIPPELA, G. et al. COVID-19 in PregnantWomen and Neonates: A Systematic Review of the Literature with Quality Assessment of the Studies. Pathogens, v. 9, n. 6, jun. 2020.

TROYER, E. A.; KOHN, J. N.; HONG, S. Are we facing a crashing wave of neuropsychiatric sequelae of COVID-19? Neuropsychiatric symptoms and potential immunologic mechanisms. Brain, behavior and immunity, v. 87, p. 34-39, jul. 2020.

TUTIYA, C. T. et al. Possible formation of pulmonary microthrombi in the early puerperium of pregnant women critically ill with COVID-19: Two case reports. Case Rep Womens Health, v. 27, e00237, jun. 2020.

VARDAVAS, C. I.; NIKITARA, K. COVID-19 and smoking: A systematic review of the evidence. Tob Induc Dis., v. 18, mar. 2020. DOI: 10.18332/tid/119324.

WANG, D. et al. Clinical Characteristics of 138 Hospitalized Patients With 2019 Novel Coronavirus-Infected Pneumonia in Wuhan, China. Journal of American Medical Asssociation, v. 323, n. 11, p. 1061-1069, fev. 2020.

YANG, J. et al. Prevalence of comorbidities and its effects in patients infected with SARS-CoV-2: a systematic review and meta-analysis. Int J Infect Dis., v. 94, p. 9195, mai. 2020.

YAO, H.; CHEN, J. H.; XU, Y. F. Patients with mental health disorders in the COVID19 epidemic. Lancet Psychiatry, v. 7, n. 4, mar. 2020.

ZHAI, P. et al. The epidemiology, diagnosis and treatment of COVID-19. International journal of antimicrobial agents, v. 55, n. 5, mai. 2020.

ZHANG, L. et al. Clinical characteristics of COVID-19-infected cancer patients: a retrospective case study in three hospitals within Wuhan, China. Ann Oncol., v. 31, n. 7, p 894-901, jul. 2020. 
Eingereicht: Juni 2021.

Genehmigt: Juni 2021. 